Recepción: 20 / 04 / 2017

Aceptación: 20 / 05 / 2017

Publicación: 15 / 06 / 2017

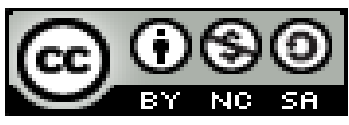

Ciencias de Producción

Artículo de Investigación

\title{
Impacto y factibilidad en la comercialización de la miel proveniente de la caña de azúcar
}

\section{Impact and feasibility in the marketing of \\ honey from sugar}

Viabilidade e impacto sobre a comercialização de

mel de cana-de-açúcar

\author{
Susana P. Morán-Rodríguez I \\ smoran@utb.edu.ec \\ Rosendo A. Gil-Avilez II \\ rgil@utb.edu.ec \\ Wendy L. Ocampo-Ulloa ${ }^{\text {III }}$ \\ wocampo@utb.edu.ec
}

Correspondencia: smoran@utb.edu.ec

I. Ingeniera Comercial; Magister en Administración de Empresas; Diploma Superior en Docencia Universitaria; Universidad Técnica de Babahoyo, Quito, Ecuador.

II. Magister Ejecutivo en Dirección de Empresas con Énfasis en Gerencia Estratégica; Diploma Superior en Investigación de la Educación a Distancia; Ingeniero Industrial; Universidad Técnica de Babahoyo, Quito, Ecuador.

III. Magister en Administración de Empresas; Ingeniera en Empresas y Administración de Negocios; Universidad Técnica de Babahoyo, Quito, Ecuador. 


\section{Resumen}

El propósito es investigar el impacto y factibilidad que tendrá la comercialización de la miel de la caña de azúcar en el Cantón Babahoyo Provincia de los Ríos, porque es uno de los productos de mayor importancia para el desarrollo comercial, por ser fuente de calorías en las dietas de todos los hogares. Este proyecto está dirigido al Cantón Babahoyo porque constituye un polo de desarrollo muy importante para el país y está encasillado dentro de la matriz productiva, por el escaso conocimiento en el mercado artesanal, en la población y la falta de investigación sobre el uso de la miel de caña de azúcar, las perspectiva de desarrollo y es importante porque va a servir de apoyo en las investigaciones que se hagan, al aumentar la producción de la miel de caña de azúcar mejoraría el posicionamiento en el mercado local, nacional e internacional incrementando su consumo, siendo una alternativa de explotación artesanal, se podría convertir en uno de los productos de exportación del Ecuador hacia otros países como medio de ingresos económicos y el sector productor de caña de azúcar ha sido un pilar importante en la economía ecuatoriana desde hace varios años. También se desarrolla una proyección del estudio técnico y financiero del consumo de miel de caña de azúcar con sus ingresos y costos de producción, la materia prima, mano de obra directa y los costos indirectos de fabricación, un plan económico financiero, resultado estudios realizados en el análisis financiero elaborado delimitando la factibilidad.

Palabras claves: Comercialización de la miel; caña de azúcar; matriz productiva; explotación artesanal. 


\begin{abstract}
The purpose is to investigate the impact and feasibility of commercialization of sugar cane honey in the Canton Babahoyo Province of the Rivers, because it is one of the products of major importance for commercial development, as a source of calories in Diets of all households. This project is aimed at the Canton Babahoyo because it constitutes a very important development pole for the country and is placed within the productive matrix, due to the lack of knowledge in the artisanal market, in the population and the lack of research on the use of honey Of sugarcane, development prospects and is important because it will serve as support in the research that is done, increasing the production of sugar cane honey would improve the positioning in the local, national and international market increasing its consumption, Being an alternative of artisanal exploitation, could become one of Ecuador's export products to other countries as a means of economic income and the sugar cane sector has been an important pillar in the Ecuadorian economy for several years. A technical and financial study of the consumption of sugar cane honey with its income and production costs, raw material, direct labor and indirect manufacturing costs, a financial economic plan, results The financial analysis elaborated delimiting the feasibility.
\end{abstract}

Key words: honey marketing; sugar cane; Productive matrix; Exploitation. 


\section{Resumo}

O objetivo é investigar o impacto ea viabilidade têm a comercialização de açúcar de cana mel em Canton Babahoyo Rios Província, porque é um dos produtos mais importantes para o desenvolvimento comercial, como uma fonte de calorias em dietas de todos os domicílios. Este projecto destina-se a Canton Babahoyo, porque é um centro de desenvolvimento muito importante para o país e é rotulado dentro da matriz produtiva, a falta de conhecimento no mercado de artesanato na população ea falta de pesquisa sobre o uso do mel cana de açúcar, a perspectiva do desenvolvimento e é importante porque dará apoio na pesquisa feita através do aumento da produção de cana-de-açúcar mel melhorar o posicionamento nos mercados locais, nacionais e internacionais, aumentando o seu consumo sendo uma mineração artesanal alternativa, poderia se tornar um dos produtos de exportação do Equador para outros países como um meio de renda e do setor de produção de cana tem sido um pilar importante na economia equatoriana por vários anos. uma projeção de estudo técnico e financeiro do consumo de cana mel com os seus rendimentos e custos de produção também desenvolve, matérias-primas, os custos trabalhistas e indiretos de fabricação diretos, um plano econômico financeira, estudos de resultados a análise financeira preparada realce a viabilidade.

Palavras-chave: omercialização de mel; cana de açúcar; matriz de produção; exploração artesanal. 


\section{Introducción.}

En la actualidad existen diversidad de pequeñas empresas en el país, las cuales emprenden el negocio con un solo propósito que es el de crecer y convertirse en grandes corporaciones a pesar de que es difícil lograr posicionarse en el mercado.

Este proyecto está diseñado para dar a conocer las ventajas y desventajas de crear nuevos productos derivados de la caña de azúcar.

La caña de azúcar es una gramínea tropical perenne con tallos gruesos y fibrosos que pueden crecer entre 3 y 5 metros de altura. Éstos contienen una gran cantidad de sacarosa que se procesa para la obtención de azúcar. La caña de azúcar es un cultivo a nivel mundial, de sus tallos se extrae la sacarosa que es uno de los primordiales suplidores nutritivos en la nutrición humana.

A su vez este cultivo permite una amplia diversidad productiva, que es mundialmente reconocida, estos productos son totalmente aprovechados, con diversos fines; económico, alimenticio, entre otros, es posible identificar amenazas en el comportamiento del mercado de la provincia de los Ríos de productos tradicionales asociados a este cultivo.

Para ser competitivos hay que implementar las tecnologías apropiadas y tomar las mejores decisiones en el momento oportuno, contando con información confiable y actualizada. La caña de azúcar, un cultivo al que se le puede procesar para obtener productos (panela, aguardiente, mieles, confites), uno de los principales derivados es el azúcar en sus diferentes tipos. 


\section{Materiales y métodos.}

Este proyecto también surge de la necesidad de que se aumente la producción de la miel de caña de azúcar en nuestro país, ya que por sus variedades en este producto, también podemos imponernos en el mercado nacional e internacional para incrementar su consumo. Siendo otra alternativa de explotación artesanal, se puede convertir en uno más de los productos de exportación del Ecuador hacia otros países como medio de ingresos económicos a nuestro País.

Además la presente información en este proyecto deja abierta oportunidades de emprendimiento de un negocio para la población, donde con un buen asesoramiento puede emprender su propio negocio donde obtendrán ingresos económicos para su hogar.

\section{Resultados.}

\section{ESTUDIO TÉCNICO}

\section{ENCUESTA SOBRE EL IMPACTO DE LA MIEL A BASE DE CAÑA DE AZÚCAR EN LA POBLACIÓN DE BABAHOYO}

La presente encuesta como objetivo fundamental el de recopilar información para saber sobre el impacto de la miel a base de caña de azúcar en la población de Babahoyo

\begin{tabular}{|l|l|}
\hline SI & 36 \\
\hline NO & 65 \\
\hline
\end{tabular}

1. ¿Conoce o ha probado la miel a base de caña de azúcar? TABLA 1 


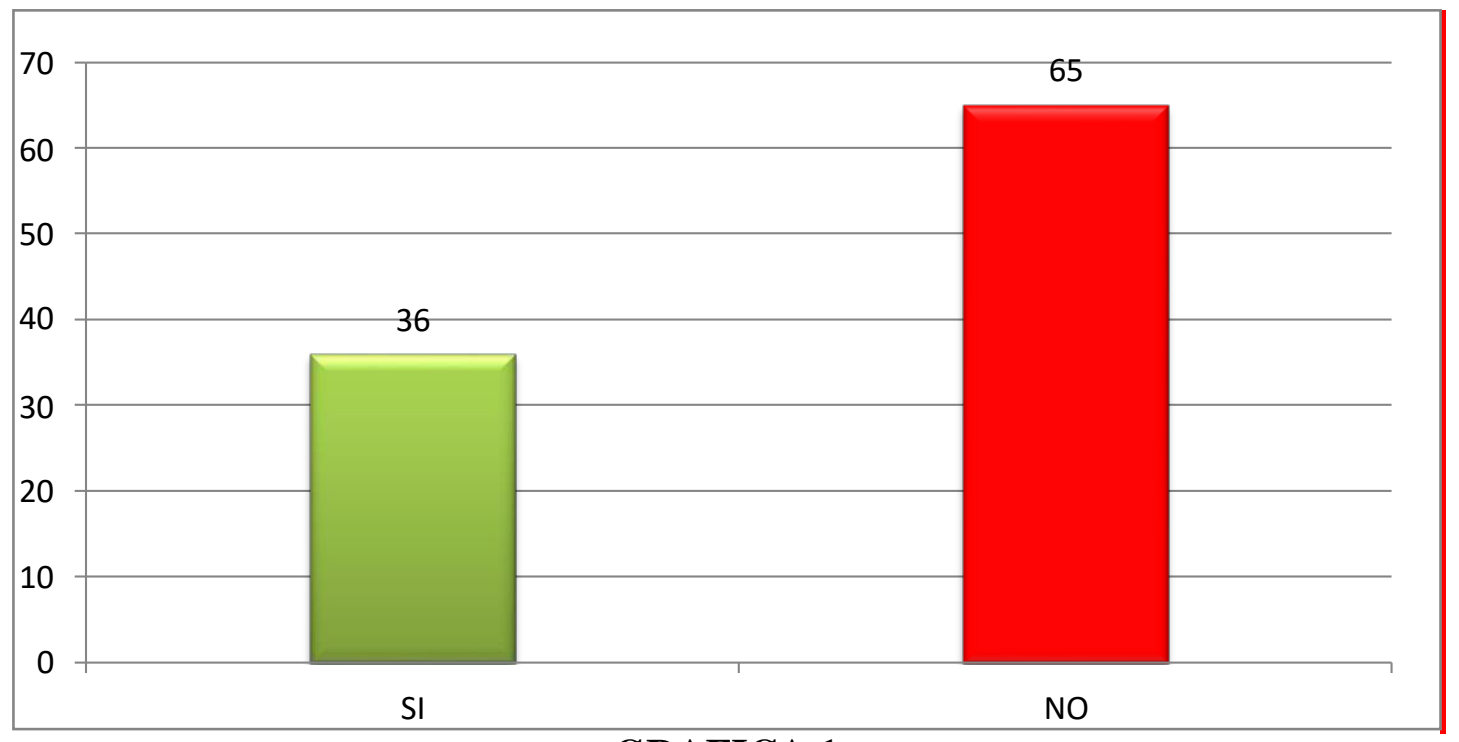

GRAFICA 1

Análisis: Aquí podemos observar que en una cantidad de 101 encuesta, hubo un 65 personas que dijeron que no y un 36 que sí han probado la miel de caña de azúcar.

\begin{tabular}{|l|l|}
\hline SI & 48 \\
\hline NO & 53 \\
\hline
\end{tabular}

2. Conoce los beneficios que la miel a base de caña de azúcar nos aporta TABLA 2

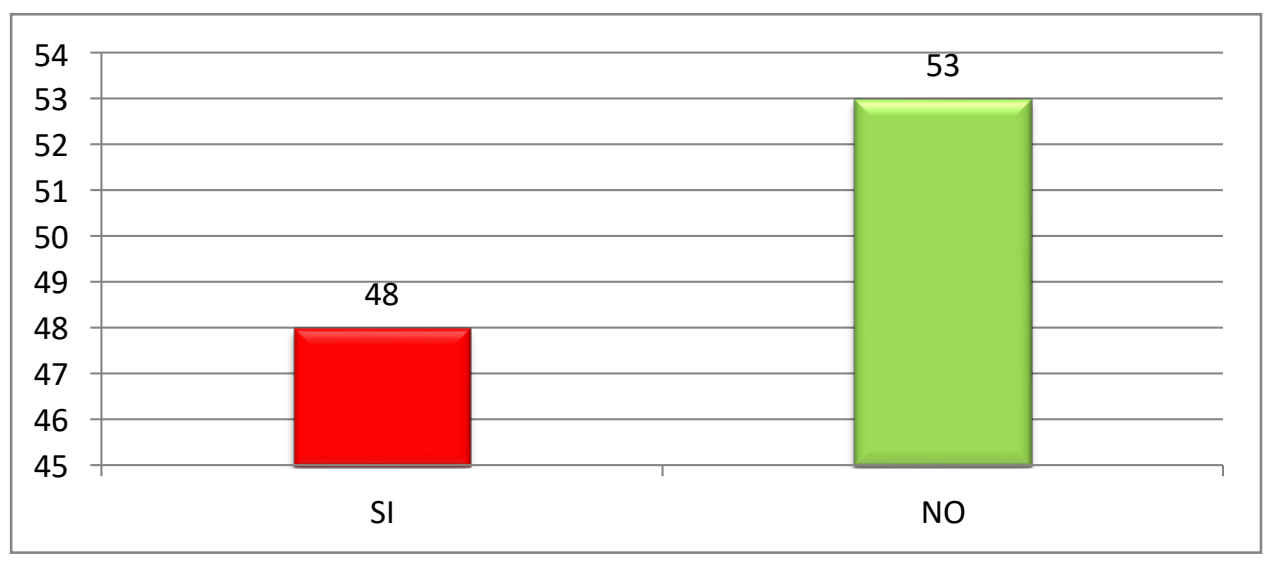

GRAFICA 2

Análisis.- En esta pregunta podemos decir que 53 personas que fueron encuesta no conocen los beneficios de la caña de azúcar y el 48 si lo conocen los beneficios que contiene la caña de azúcar. 


\begin{tabular}{|r|r|}
\hline Si & 20 \\
\hline No & $\mathbf{8 1}$ \\
\hline
\end{tabular}

\section{3. ¿Le gustaría probar?}

\section{TABLA 3}

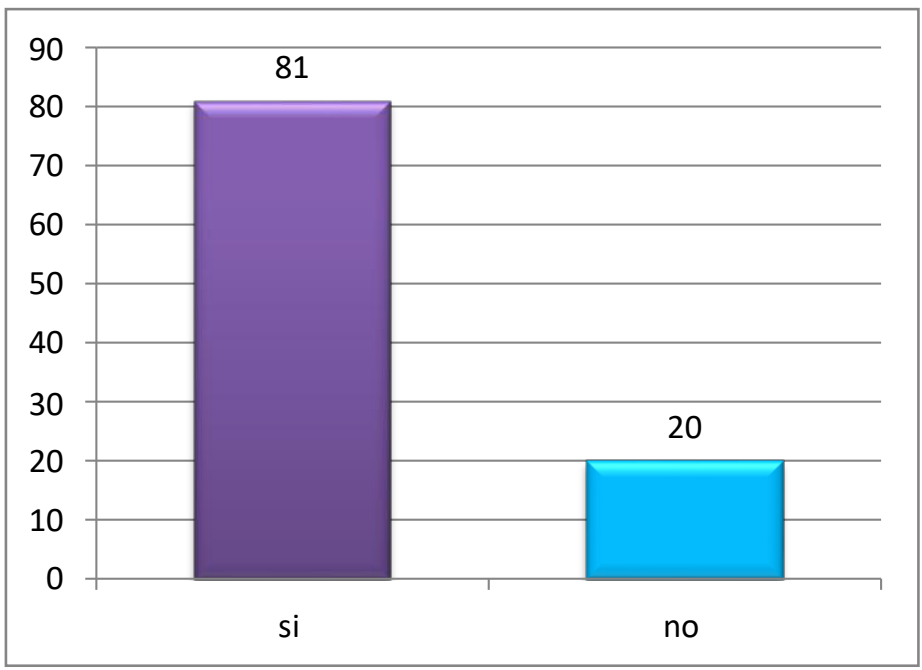

GRAFICA 3

Análisis.- En esta pregunta de que si les gustaría probar la miel de caña de azúcar hubo un 81 de los encuestado que dijeron que si y un 20 que no, esto nos indica que nuestro proyecto si es factible porque hubo un mayor porcentaje que dijo que sí.

\begin{tabular}{|l|l|}
\hline 1 & 7 \\
\hline 2 & 13 \\
\hline 3 & 20 \\
\hline 4 & 27 \\
\hline 5 & 34 \\
\hline
\end{tabular}

4. En la escala de 1 al 5 donde el 5 es muy interesante y 1 es nada interesante ¿Cómo califica la miel hecha a base de caña de azúcar?

TABLA 4 


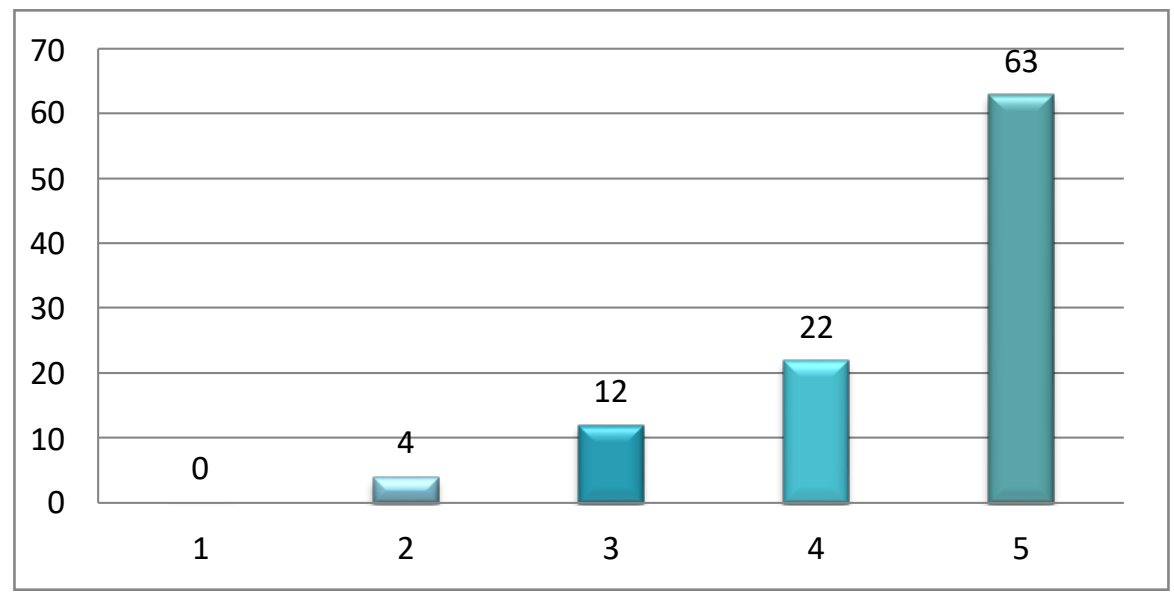

GRAFICA 4

Análisis.- El número de encuestado que respondió el nivel 5 que es muy interesante es del 63 y el que escogió el nivel 4 con 22, y el nivel 3 es de 12, nivel 2 son 4, y el nivel 1 es de 0 personas. Con esto podemos decir que la opción más factibles es el nivel 5 de 63 que muy interesante este producto.

\begin{tabular}{|l|l|}
\hline Sus beneficios & 53 \\
\hline Su sabor & 33 \\
\hline Ninguno & 14 \\
\hline Otros & 1
\end{tabular}

5. Cual o cuales de los siguientes aspectos le atraen del producto. TABLA 5

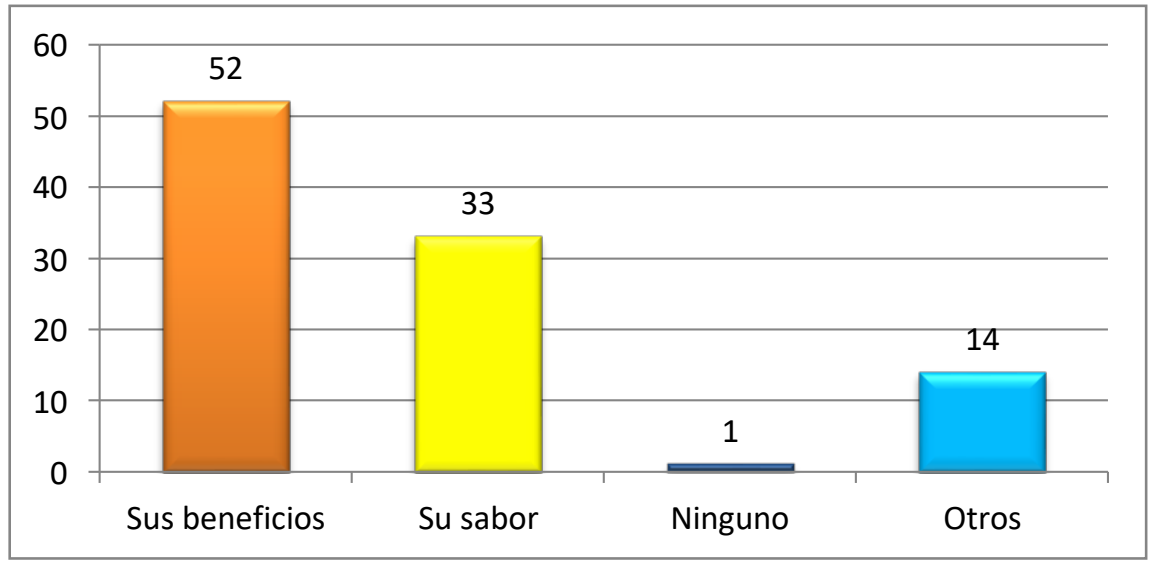

GRAFICA5 
Análisis.- Como podemos observar en el gráfico de barra que 52 personas le gusta los beneficios de la miel de la caña de azúcar, y 33 persona le gusta el sabor, a 14 personas otros y 1 ninguno

\begin{tabular}{|c|c|}
\hline Mercado & 30 \\
\hline Tiendas de barrio & 12 \\
\hline supermercados & 59 \\
\hline
\end{tabular}

6. En qué lugar le gustaría comprar les gustaría este producto

TABLA 6

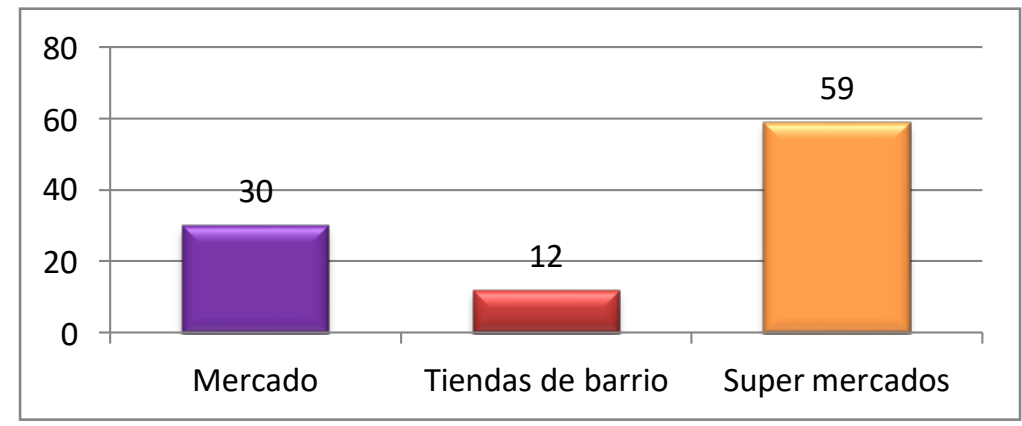

GRAFICA 6

Análisis.- en esta pregunta los encuestadores dijeron 59 que le gustaría comprar el producto en los supermercados, el mercado 30 persona y en las tiendas del barrio 12 persona

\begin{tabular}{|l|l|}
\hline Siempre & 45 \\
\hline Con frecuencia & 33 \\
\hline A veces & 8 \\
\hline Nunca & 15 \\
\hline
\end{tabular}

\section{Con qué frecuencia consumirá este producto} TABLA 7 


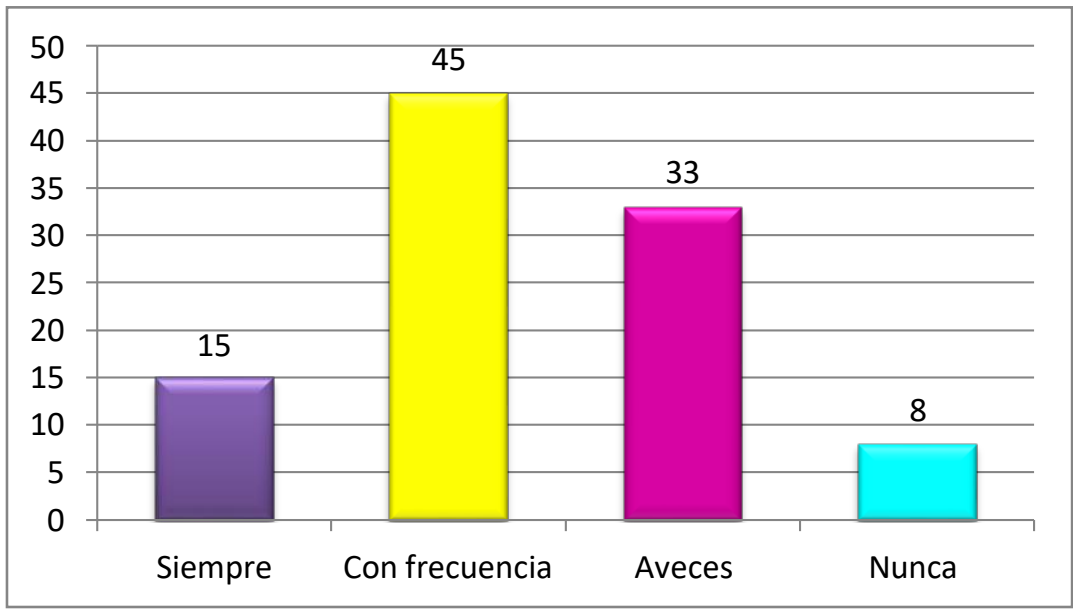

GRAFICA 7

Análisis: Como se observa en el gráfico de barra, podemos decir 45 de las personas lo consumirá con frecuencia, 33 personas lo consumirá a veces, y mientras que un siempre lo consumirá 15 personas, y 8 persona nunca lo haría en consumir este producto.

\section{Plaza}

También llamada distribución, es la medio que permite que el producto llegue al consumidor, lugar y al tiempo adecuado.

La empresa comercializará el producto en el cantón Babahoyo

Por lo cual su medio de distribución indirecto, es decir utilizando un intermediario o detallista, ya que un canal de distribución directo.

Existen beneficios que los intermediarios aportan para la empresa como son los siguientes:

Los detallistas transportan y asumen el riesgo, en caso que exista algún contratiempo con la mercadería.

Se encargan de asignar un lugar y el tiempo adecuado para el producto. 
Susana P. Morán-Rodríguez; Rosendo A. Gil-Avilez; Wendy L. Ocampo-Ulloa

Al estar en contacto directo con el consumidor final, recomiendan a la empresa las cantidades óptimas a producir.

Los intermediarios ayudan a las empresas a surgir son principalmente distribuidores autorizados, mayoristas, detallistas.

\section{Canales de Distribución}

Es un conjunto de organizaciones que dependen entre sí y que participa en el proceso de llevar el producto a la disposición del consumidor.

Para este plan se ha designado un canal de distribución indirecto, donde quienes intervienen son el Fabricante-Detallista-Consumidor Final, debido a los detallistas tienen relaciones ya creadas en el mercado para poner de venta en el lugar de expendio el producto.

\section{Plan de Marketing}

Es crear y mantener con las estrategias y metas, capacidades de una organización con sus oportunidades en el marketing.

Tener clara misión en la empresa y objetivos de apoyo diseñando cartera de negocios y coordinar funciones.

El plan en el marketing pretende diagnostico externa e interna en la empresa con el propósito de objetivos claros preparados en situaciones presente externa e interna para alcanzar metas de las mismas. 


\section{Misión}

Ser líder en la comercialización de una miel a base de lo natural como es la caña de azúcar, el cual proporciona muchos beneficios para la salud, satisfaciendo a los consumidores con algo nuevo de calidad.

Además de tener los objetivos bien claros, de lo que deseamos alcanzar con el mismo.

\section{Visión}

Posesionarnos en los diferentes mercados nacional e internacional, siendo el único o principal productor de dicha miel, siempre y cuando satisfaciendo las necesidades de los clientes para poder introducir el producto en el mercado.

El que se lograra con la acogida que se tenga por parte consumidores.

\section{FODA}

\begin{tabular}{|l|l|l|l|}
\hline \multicolumn{1}{|c|}{ Amenaza } & \multicolumn{1}{|c|}{ Fortaleza } & \multicolumn{1}{c|}{ Debilidad } & Problema \\
\hline $\begin{array}{l}\text { No conocer el } \\
\text { beneficio de la }\end{array}$ & Es bueno para la & La falta de agua & Si no hay \\
\hline miel de la caña & para la & suficiente agua \\
\hline de azúcar. & producción & los fertilizantes \\
\hline
\end{tabular}




\begin{tabular}{|c|c|c|c|}
\hline $\begin{array}{l}\text { No tener } \\
\text { suficiente } \\
\text { acogida en el } \\
\text { mercado. }\end{array}$ & $\begin{array}{l}\text { Dar el debido } \\
\text { conocimiento de } \\
\text { las personas para } \\
\text { la elaboración de } \\
\text { la miel de caña } \\
\text { de azúcar. }\end{array}$ & $\begin{array}{l}\text { No tener una } \\
\text { liquidez } \\
\text { monetaria. }\end{array}$ & $\begin{array}{l}\text { El trabajo en } \\
\text { pequeñas } \\
\text { superficies eleva } \\
\text { considerablemente } \\
\text { los costos de } \\
\text { operación }\end{array}$ \\
\hline $\begin{array}{l}\text { No contar con } \\
\text { la suficiente } \\
\text { estructura. }\end{array}$ & $\begin{array}{l}\text { El producto es } \\
\text { natural. }\end{array}$ & $\begin{array}{l}\text { Que tenga } \\
\text { acogida en el } \\
\text { mercado. }\end{array}$ & \\
\hline $\begin{array}{l}\text { No conocer la } \\
\text { situación } \\
\text { económica. }\end{array}$ & $\begin{array}{l}\text { Tener } \\
\text { maquinarias que } \\
\text { son de poca } \\
\text { inversión. }\end{array}$ & $\begin{array}{l}\text { Producto para el } \\
\text { consumo } \\
\text { humano. }\end{array}$ & \\
\hline
\end{tabular}

TABLA8

ESTUDIO TÉCNICO

\section{Proyección De La Oferta}

\section{0 litros}

Incremento de $10 \%$

Empezara producir con un $50 \%$

\section{Capacidad del Proyecto}




\begin{tabular}{|l|l|l|}
\hline AÑOS & $\begin{array}{l}\text { CAPTACIÓN } \\
\text { MERCADO }\end{array}$ & $\begin{array}{l}\text { CAPTACION DEL } \\
\text { PROYECTO (LITROS) }\end{array}$ \\
\hline 1 & $60 \%$ & 33348 \\
\hline 2 & $70 \%$ & 38906 \\
\hline 3 & $80 \%$ & 44464 \\
\hline 4 & $90 \%$ & 50022 \\
\hline 5 & $100 \%$ & 55580 \\
\hline
\end{tabular}

TABLA9

Cuadro De Consumo De Miel De Caña De Azúcar Según Encuestas

Primer año se necesitará producir $\mathbf{3 3 . 3 4 8}$ litros de miel de caña de azúcar

\begin{tabular}{|l|l|l|l|}
\hline Concepto & $\begin{array}{l}\text { Valor } \\
\text { Total } \\
\text { (USD) }\end{array}$ & Unitario/litros & Unitario/500ml \\
\hline Costo de Producción & $76.750,50$ & 2,30 & 1,15 \\
\hline Gastos de Administración & $17.304,00$ & 0,52 & 0,26 \\
\hline Gasto de Ventas & 15200,00 & 0,46 & 0,23 \\
\hline Gasto Financiero & $18.310,00$ & 0,55 & 0,28 \\
\hline $\begin{array}{l}\text { Total de Costos y Gastos } \\
\text { UTILIDAD FIJDA }\end{array}$ & $127.564,50$ & 3,83 & 1,92 \\
\hline (30\%) & & $\mathbf{1 , 1 5}$ & $\mathbf{0 , 5 8}$ \\
\hline PRECIO PROPUESTO & & $\mathbf{4 , 9 8}$ & $\mathbf{2 , 5 0}$ \\
\hline
\end{tabular}

\section{Cuadro De Determinación De Precio De Venta}

TABLA 10

\section{Conclusiones.}

En conclusión este proyecto de impacto y factibilidad de elaboración de la caña de azúcar en el Cantón Babahoyo de la provincia de Los Ríos se enfoca en esta dicha población donde intervienen personas de ambos sexos que son los involucrados para consumir este producto. En 
donde se busca conocer si el producto tendrá rentabilidad en el mercado una vez posesionado en el mismo, ya que la caña de azúcar en el Ecuador es una producción muy buena que inclusive también se la exporta a diferentes lugares.

Siendo el Ecuador uno de los que produce la azúcar para el consumo nacional, por tal instancia nuestro propósito es que las personas del mencionado Cantón consuman la miel de caña de azúcar ya que es un producto agrícola extraído de forma natural que contiene muchos nutrientes beneficiosos para la salud de las personas.

Puesto que se realizó un estudio de mercado en la población en donde podemos identificar que la miel de caña de azúcar si va tener una aceptabilidad por la población debido a sus propiedades que contiene este producto, ya que es saludable para el consumo diario.

\section{Agradecimiento}

Doy gracias a Dios, en primer lugar, luego a mis padres por su apoyo incondicional, a mi Tutor en el que siempre encontré una guía y a todas las personas que directa o indirectamente contribuyeron en que avance y culmine el presente trabajo, especialmente a esa personita especial que me apoyó durante todo el desarrollo del mismo.

\section{Bibliografía.}

BioEnciclopedia. (1 de julio de 2016). BioEnciclopedia. Obtenido de http://www.bioenciclopedia.com/canade-azucar/

Castillo, R. O. (s.f.). El Productor. Obtenido de http://elproductor.com/2012/07/04/cana-de-azucar-cultivopara-la-sostenibilidad/

Linnaeus, C. v. (s.f.). EcuRed. Obtenido de https://www.ecured.cu/Ca\%C3\%B1a_de_az\%C3\%BAcar

MAYRA SOFÍA CASARES ACOSTA. (2010). Obtenido de http://repositorio.ute.edu.ec/bitstream/123456789/2036/1/41895_1.pdf 
SARMIENTO, J. P. (s.f.). ecuaquimica. Obtenido de https://www.ecuaquimica.com.ec/info_tecnica_cana.pdf

Pearson Edicion.

VIEJO OJEDA KARINA ELIZABETH. (2013). Obtenido de http://cia.uagraria.edu.ec/archivos/VIEJO\%20OJEDA\%20KARINA\%20ELIZABETH.pdf

Cubillo, J. (1997). La inteligencia empresarial en las pequeñas y medianas empresas competitivas de América Latina - algunas reflexiones. Brasilia: Scielo.

México, A. d. (2013). Toluca-Mexico|: Redalyc.

Moreno-Moya, M., \& MunueraAlemán2, J. L. (2014). ¿Es importante la creatividad en el desarrollo de nuevos productos? Madrid-España: Redalyc.

Noé Aguilar Rivera*, G. G. (2011). Factores de competitividad de la agroindustria de la caña de azúcar en México. Hermosillo: Redalyc.

Rivera, N. A. (2010). La caña de azúcar y sus derivados en la huasteca San Luís Potosí México. Costa Rica: Redalyc. 